\title{
Clinical and laboratory characteristics associated with a high optical density anti-platelet factor 4 ELISA test
}

\author{
This article was published in the following Dove Press journal: \\ Journal of Blood Medicine \\ 19 November 2015 \\ Number of times this article has been viewed
}

Purpose: Diagnosing heparin-induced thrombocytopenia, a potentially catastrophic immunemediated disorder, continues to pose significant challenges for clinicians, as both clinical and laboratory tools lack specificity. There is mounting evidence supporting a positive correlation between definitive heparin-induced thrombocytopenia and optical density (OD) positivity from the widely available anti-platelet factor 4 enzyme-linked immunosorbent assays (PF4 ELISAs). However, the clinical features distinguishing these patients remain poorly understood.

Patients and methods: To better characterize this group, we conducted a case-controlled, retrospective chart review of patients from two large, urban academic institutions who underwent a PF4 ELISA at a central laboratory. Associations between OD and 18 clinical characteristics were calculated using the Fisher's exact test for categorical variables and Wilcoxon rank-sum test for continuous variables.

Results: In total, 184 negative patients $(\mathrm{OD}<0.7)$, and 121 positive patients (OD $>0.7)$, including 74 low-positive patients $(0.7<\mathrm{OD}<1.4)$ and 47 high-positive patients $(\mathrm{OD}>1.4)$ were identified. Several clinical variables were significantly different in the negative group compared with the positive group, including hospital day $(P<0.001)$, previous admission within the past 3 months $(P<0.001)$, and the presence of a new thrombus $(P=0.003)$. However, many of these variables were not different between the negative and low-positive group, and were only distinct between the negative and high-positive group. When the low-positive and high-positive groups were compared, only the 4T score was significantly different $(P=0.003)$.

Conclusion: These data indicate that those with OD $>1.4$ form a distinct clinical group and support the clinical utility of the $4 \mathrm{~T}$ score.

Keywords: heparin-induced thrombocytopenia, platelet factor 4 ELISA, thrombocytopenia, HIT, heparin

\section{Introduction}

Heparin-induced thrombocytopenia (HIT) is a potentially severe, adverse reaction to the widely used anticoagulant heparin. Although heparin is a well-established effective anticoagulant, in the presence of heparin, auto IgG antibodies can bind to epitopes on platelet factor 4 (PF4; released from the alpha granules of platelets) and activate platelets via the FcgRII receptors on the platelet's surface to induce a thrombotic cascade. Multiple studies suggest that HIT occurs in up to $3 \%$ of hospitalized patients exposed to unfractionated heparin, and up to a third of these patients can develop lifethreatening thrombosis. ${ }^{1-4}$ Because HIT is treated by ceasing heparin administration and administering an alternative form of anticoagulation, the rapid and accurate diagnosis of HIT has important clinical implications.
Correspondence: Lawrence B Gardner Department of Medicine, NYU Langone Medical Center, NYU School of Medicine, 550 First Avenue, New York, NY 10016, USA Email lawrence.gardner@nyumc.org 
Currently, there is no universally accepted gold standard for the diagnosis of HIT, but common clinical practice includes both clinical and laboratory findings. ${ }^{5,6}$ Useful clinical findings include a drop in platelet count by $50 \%$ or below $150,000 / \mu \mathrm{L}$, a fall in platelet count starting over 5 days after heparin exposure in a heparin naïve patient, and the absence of other factors (such as infection and other drug use) that could lead to thrombocytopenia. In an effort to improve the ability to accurately predict and standardize the diagnosis of HIT, a weighted scoring system, which incorporates four typical features of HIT including magnitude of thrombocytopenia, timing of thrombocytopenia, presence of thrombosis or other sequelae of HIT, and the likelihood of other causes of thrombocytopenia, has been commonly utilized. ${ }^{7-11}$ This " $4 \mathrm{~T}$ " score has been noted to have a high sensitivity for the diagnosis of HIT but is non-specific. ${ }^{7,12}$

Additionally, the presence or absence of anti-heparin/PF4 antibodies has been used to assist in the diagnosis of HIT. ${ }^{13-15}$ These antibodies, which have been demonstrated to cause thrombocytopenia in association with PF4 and heparin, are present in almost all patients who are diagnosed with HIT. ${ }^{15,16}$ However, while the absence of these antibodies is thought to have a negative predictive value for the diagnosis of HIT of over $90 \%$, the presence of HIT antibodies has been estimated to have as low as a $5 \%$ positive predictive value in select patient populations. ${ }^{6,17-20}$ While some studies suggest that the serotonin release assay (SRA) has a higher specificity than the more commonly used PF4 enzyme-linked immunosorbent assay (PF4 ELISA) technique, ${ }^{21-23}$ the SRA is technically demanding, and typically available only in reference laboratories, which can result in delayed results, and is still positive in the absence of many of the clinical findings associated with HIT. ${ }^{19,24-27}$ In fact, though many rely on the SRA during the diagnosis of HIT, this positive predictive value of this test can range from $5 \%$ to $50 \%$ depending on the patient population. ${ }^{22}$ Despite the limitations of techniques that detect the presence of HIT antibodies, there is mounting evidence supporting a positive correlation between PF4 ELISA optical density (OD) positivity, the likelihood of a positive SRA, and the presence of thrombosis. ${ }^{18,28-31}$ Thus, a high OD positivity associates with a higher likelihood of true HIT. Nonetheless, the clinical and laboratory characteristics that distinguish patients with high OD values remain poorly understood.

Inappropriately diagnosing HIT can have multiple serious consequences, including the cessation of heparin, the deferment of procedures requiring heparin, the initiation of costly and potentially dangerous other anticoagulants, and the failure to correctly identify the actual cause of thrombocytopenia..$^{3,32-38}$ The lack of specificity of both clinical features of HIT and the presence of HIT antibodies highlight the need to improve the accuracy of diagnostic methods to facilitate appropriate empiric treatment. We, therefore, examined specific clinical and laboratory data from all patients at two large, urban academic hospitals who had PF4 ELISAs performed at a central laboratory over a 3-year period to determine if any readily available clinical and/or laboratory finding associated with a high PF4 ELISA OD.

\section{Patients and methods Patients and PF4 ELISA testing}

This case-control, retrospective study was reviewed and approved by the Institutional Review Board at NYU Langone Medical Center. Because of the retrospective nature of this study this Review Board did not require patient consent. A laboratory database was used to identify all hospitalized patients who had PF4 ELISA at the Tisch Hospital and Bellevue Hospital Center between July 1, 2009 and July 1, 2014. PF4 ELISA testing was performed using the LIFECODES PF4 Enhanced assay kit (Immucor GTI Diagnostics, Inc., Waukesha, WI, USA) according to the manufacturer's guidelines and validated with internal controls. The assay is a polyimmunoglobulin assay (anti-IgG/A/M) utilizing 60 units of heparin in the positive control. Internal positive and negative control values were recorded for each patient's PF4 ELISA. Testing for anti-heparin/ PF4 antibodies was performed at the discretion of each patient's medical team. For patients who had more than one PF4 ELISA performed, only the initial result was considered.

\section{Chart review}

Inclusion criteria included PF4 ELISA tests from January to July 2013 and all "positive" values from July 2009 to July 2014. Additionally, all included patients were older than 18 years and had full clinical data in the medical record. No direct contact with patients was made. Two trained abstracters collected data for each patient including age, admitting service, hospital day at the time of the assay, days since first heparinization, white blood cell count, hemoglobin, platelet count at presentation and at the time of the assay, creatinine, international normalized ratio, thrombosis at the time of the assay, antibiotics at the time of thrombocytopenia, positive blood culture, surgical procedure within the last 2 weeks, cardiothoracic surgery within the last 2 weeks, hospital admission within the last 3 months, and death during the current hospitalization. Laboratory data were recorded at the initial presentation and/or at the time of the PF4 ELISA assay. All thromboses were radiographically confirmed. Probably, thromboses reflect those thromboses that were incidentally 
found with an unclear duration and/or relationship to the HIT diagnosis. Values calculated from the collected clinical data included the percentage drop in platelet count from admission and the 4T score. Because of the previously mentioned limitations of the SRA,22 and the fact that only a small fraction of patients were tested for antibodies using the SRA precluding robust statistical analyses, we do not report on the results of these tests.

\section{Statistical analyses}

The Wilcoxon rank-sum test was used to evaluate continuous variables. Pearson's $\chi^{2}$ tests were performed to evaluate categorical variables for statistical differences. Fisher's exact test was used to evaluate categorical data with small sample sizes. Two-way analysis of variance testing was used to determine OD differences between different clinical probability groups. Pearson correlation values were calculated to determine linear regression. A $P$-value $<0.05$ was considered statistically significant.

\section{Results}

In order to define "high-positive" and "low-positive" PF4 ELISA OD thresholds, we first analyzed all patients who had a PF4 ELISA test between January 1, 2013 and July 1, 2013. There was a wide range of "positive" OD readings above our institutional accepted, manufacturer suggested, a positive threshold of 0.7 OD (Figure 1A). We defined the high-positive OD group as those with ODs exceeding the second quartile. Based on these analyses, negative values were defined as an OD $<0.7$, low-positive tests were defined as $0.7 \leq \mathrm{OD} \leq 1.4$, and high-positive tests were defined as OD $>1.4$. Of note, these subgroups correlated well with the prevalence of HIT reported in the prior studies. ${ }^{39,40}$ Subsequent analyses compared the low-positive and highpositive groups over a 4-year period using the thresholds defined above and those with negative values in 2013.

In total, 305 unique patients met inclusion criteria. These included 184 patients with a negative PF4 ELISA (OD $<0.7$ ), 74 patients with a low-positive PF4 ELISA $(0.7 \leq \mathrm{OD} \leq 1.4)$, and 47 patients with a high-positive PF4 ELISA (OD >1.4). Our cohort included patients from multiple services including medicine $(31.6 \%)$, surgery (44.9\%), and intensive care (12.8\%). Of the patients cared for by surgical teams, $52.8 \%$ were admitted to the cardiothoracic service. There were no significant differences in the PF4 ELISA OD distribution based on primary teams between patient groups (Figure 1B). Likewise, ODs were not significantly different for other demographic criteria, such as age and recent surgical procedures.

\section{A}

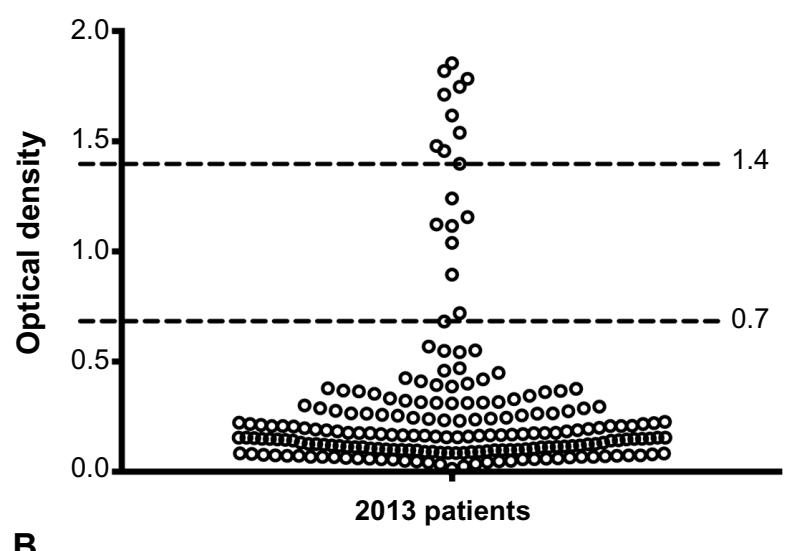

B

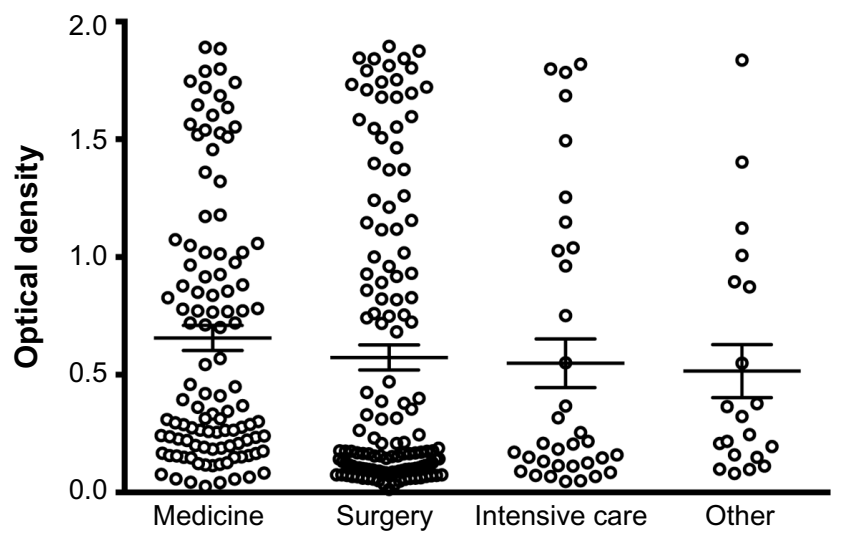

Figure I Anti-heparin/platelet factor 4 enzyme-linked immunosorbent assay (PF4 ELISA) optical density (OD) values in a cohort of patients.

Notes: (A) Scatterplot demonstrating the distribution of OD of all patients receiving anti-heparin/PF4 ELISAs in 2013. Dashed lines represent the threshold values distinguishing patients considered to have positive $(O D>0.7)$ and high-positive (OD $>$ I.4) PF4 ELISA tests. (B) Scatterplot demonstrating the distribution of the OD of all patients receiving anti-heparin/PF4 ELISAs based on admitting service. Mean OD and the standard error of the mean are represented by the horizontal error bars.

We then compared clinical variables between those with negative assays $(\mathrm{OD}<0.7)$ and those with any positive PF4 ELISA (OD $\geq 0.7)$. Those with a positive test had been in the hospital longer at the time the assay was taken than those in the negative test $(P<0.001$; Table 1$)$. The patients with positive PF4 ELISA did not have a significantly different platelet count than those with negative PF4 ELISA. However, those with positive PF4 ELISA were significantly more likely to demonstrate signs of acute inflammation or infection, such as leukocytosis $(P=0.026)$ and antibiotic therapy $(P=0.006)$ and to have an onset of thrombocytopenia 5-10 days after the initiation of heparin $(P<0.001)$ (Table 2$)$. The patients with positive tests were also more likely to have a previous admission in the preceding 3 months $(P=0.001)$ (Table 1$)$ and were more likely to have a new thrombosis at the time of the assay $(P=0.003)$ (Table 2). No mortality difference was found between negative and positive groups. 
Table I Clinical characteristics of patients included in the study

\begin{tabular}{|c|c|c|c|c|c|c|c|}
\hline \multirow[t]{2}{*}{ Demographics } & \multirow{2}{*}{$\begin{array}{l}O D<0.7 \\
(n=184)\end{array}$} & \multirow{2}{*}{$\begin{array}{l}0.7 \leq O D \leq 1.4 \\
(n=74)\end{array}$} & \multirow{2}{*}{$\begin{array}{l}O D>1.4 \\
(n=47)\end{array}$} & \multicolumn{4}{|l|}{$P$-value } \\
\hline & & & & - vs + & - vs low + & - vs high + & Low + vs high + \\
\hline Age in years*, median (range) & $66(17-100)$ & $65(25-92)$ & $72(10-93)$ & NS & NS & NS & NS \\
\hline Hospital day*, median (range) & $5(0-40)$ & $10(1-120)$ & $12(5-102)$ & $<0.001$ & $<0.001$ & $<0.001$ & NS \\
\hline Surgery within 2 weeks** & $100(54.1 \%)$ & $30(45 \%)$ & $26(55 \%)$ & NS & NS & NS & NS \\
\hline Cardiac surgery within 2 weeks** & $53(28.7 \%)$ & $15(22 \%)$ & $16(34 \%)$ & NS & NS & NS & NS \\
\hline Admission within last 3 months** & $65(35.5 \%)$ & $36(54 \%)$ & $27(57 \%)$ & 0.001 & 0.010 & 0.007 & NS \\
\hline Death during current hospitalization** & $25(13.6 \%)$ & II (I6\%) & $3(7 \%)$ & NS & NS & NS & NS \\
\hline \multicolumn{8}{|l|}{ Service* } \\
\hline Medicine & $60(32.6 \%)$ & $19(28.3 \%)$ & 15 (3I.9\%) & & & & \\
\hline Surgery & 86 (46.7\%) & 26 (38.8\%) & 22 (46.8\%) & & & & \\
\hline Cardiac surgery & 50 (27.2\%) & 13 (19.4\%) & 12 (25.5\%) & & & & \\
\hline Intensive care & 22 (II.6\%) & 7 (I0.4\%) & $4(8.5 \%)$ & & & & \\
\hline
\end{tabular}

Notes: *Wilcoxon rank-sum test, **Pearson's $\chi^{2}$, not significant (NS; $\left.P>0.05\right)$. $-=$ negative test $(\mathrm{OD}<0.7)$; low $+=0.7 \leq \mathrm{OD} \leq \mathrm{I} .4$; high $+=\mathrm{OD}>\mathrm{I} .4$.

Abbreviation: OD, optical density.

We next subcategorized patients with a positive PF4 ELISA into those with low-positive $\mathrm{OD}(0.7 \leq \mathrm{OD} \leq 1.4)$ and those with high-positive OD (OD $>1.4)$. Compared with the negative group, those with a low-positive OD also had the PF4 ELISA sent later in their hospitalization $(P<0.001)$ and were more likely to have had a previous admission within the past 3 months (Table 1). They were also more likely to have the onset of thrombocytopenia occur 5-10 days after heparin exposure, but did not have a significantly different platelet count than the negative assay group (Table 2).

Analyses comparing the high-positive group (OD $>1.4$ ) to the negative group $(\mathrm{OD}<0.7)$ revealed several differences that were not noted when the low-positive group was compared to the negative group. In fact, many of the differences noted between the positive and negative groups, including white blood cell count, use of antibiotics, and thromboses could be attributed entirely to those patients in the high-positive OD group. The high-positive OD group also had a larger drop in platelet count ( $P=0.019$ ) (Table 2 ) compared with the negative group, and a significantly increased prevalence of thromboses. Additionally, there was a statistical trend toward decreased mortality during the current hospitalization in the high-positive group.

No single clinical characteristic significantly differed between the high-positive group and the low-positive

Table 2 Laboratory characteristics of patents included in the study

\begin{tabular}{|c|c|c|c|c|c|c|c|}
\hline \multirow[t]{2}{*}{ Laboratory values } & \multirow{2}{*}{$\begin{array}{l}\text { OD }<0.7 \\
(n=184)\end{array}$} & \multirow{2}{*}{$\begin{array}{l}0.7 \leq O D \leq I .4 \\
(n=74)\end{array}$} & \multirow{2}{*}{$\begin{array}{l}O D>1.4 \\
(n=47)\end{array}$} & \multicolumn{4}{|l|}{$P$-value } \\
\hline & & & & $-v s+$ & - vs low + & - vs high + & Low + vs high + \\
\hline $\begin{array}{l}\text { White blood cell count*, } \\
\text { median (range) }\end{array}$ & $9.3(2.5-43.6)$ & $10.8(2.0-24.6)$ & II.I (2.I-34.5) & 0.026 & NS & 0.045 & NS \\
\hline Hemoglobin*, median (range) & $9.8(6.1-19.9)$ & $10(7-15.2)$ & $9.6(6.3-16.3)$ & NS & NS & NS & NS \\
\hline $\begin{array}{l}\text { Platelet count at time of } \\
\text { assay*, median (range) }\end{array}$ & $82(\mid 7-351)$ & $96(6-742)$ & $76(16-384)$ & NS & NS & NS & NS \\
\hline Creatinine*, median (range) & $1.0(0.2-12.2)$ & I.2(0.2-8.6) & $1.0(0.5-8.8)$ & NS & NS & NS & NS \\
\hline $\begin{array}{l}\text { International normalized } \\
\text { ratio*, median (range) }\end{array}$ & $1.30(0.80-11.09)$ & $1.27(0.99-4.60)$ & $1.28(0.95-4.97)$ & NS & NS & NS & NS \\
\hline Antibiotics** & $80(43.2 \%)$ & $38(58 \%)$ & $30(64 \%)$ & 0.006 & 0.053 & 0.014 & NS \\
\hline Positive blood culture** & $15(8.2 \%)$ & $9(13 \%)$ & $8(17 \%)$ & 0.072 & NS & 0.074 & NS \\
\hline 4T score***, median (range) & $3(0-7)$ & $3(0-7)$ & $4(I-7)$ & $<0.001$ & $0.04 I$ & $<0.001$ & 0.003 \\
\hline $\begin{array}{l}\text { Onset } 5-10 \text { days after } \\
\text { heparin exposure** }\end{array}$ & $55(29.7 \%)$ & $32(48 \%)$ & $28(60 \%)$ & $<0.001$ & 0.007 & $<0.001$ & NS \\
\hline $\begin{array}{l}\text { Drop in platelet count from } \\
\text { admission (\%), median (range) }\end{array}$ & $46(91$ to 465$)$ & 44 (100 to 207$)$ & $55(93$ to 321$)$ & NS & NS & 0.019 & 0.067 \\
\hline Thrombosis at time of assa & & & & & & & \\
\hline New & $8(4.3 \%)$ & $9(13 \%)$ & $9(19 \%)$ & 0.003 & 0.055 & 0.002 & NS \\
\hline Possible & II (6.0\%) & $3(4 \%)$ & $4(9 \%)$ & & & & \\
\hline No & $166(89.7 \%)$ & 55 (82\%) & 34 (72\%) & & & & \\
\hline
\end{tabular}

Notes: *Wilcoxon rank-sum test, ***earson's $\chi^{2}$ test, ***Fisher's exact test, not significant $(\mathrm{NS} ; \mathrm{P}>0.05) .-=$ negative test $(\mathrm{OD}<0.7)$; low $+=0.7 \leq \mathrm{OD} \leq 1.4$; high $+=\mathrm{OD}>\mathrm{I.4}$. Abbreviation: OD, optical density. 
group. However, remarkably, the 4T score $(P=0.003)$ was significantly higher in the high-positive PF4 ELISA OD group than the low-positive PF4 ELISA group. The 4T score was also significantly increased in all patients with positive PF4 ELISA when compared with negative patients, and significant $(P=0.041)$ (Table 2$)$ even when only the low-positive PF4 ELISA group was compared with the negative group. Further analyses demonstrated a direct correlation between $4 \mathrm{~T}$ score and OD result (Figure 2A). When the patients were grouped based on $4 \mathrm{~T}$ score interpretations (low $<4$, intermediate $4-5$, and high $>5$ clinical probability scores), there was an increasing mean OD: low $0.498 \pm 0.50$, intermediate $0.689 \pm 0.65$, and high $1.28 \pm 0.65$ (Figure 2B). Additional numerical corrections, including OD normalization to internal positive and negative control OD values, failed to improve the Pearson's correlation value (data not shown).

A

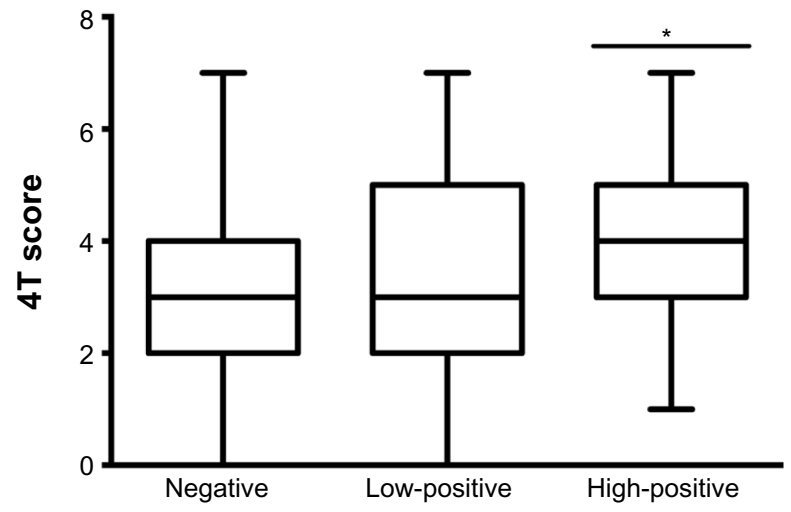

B

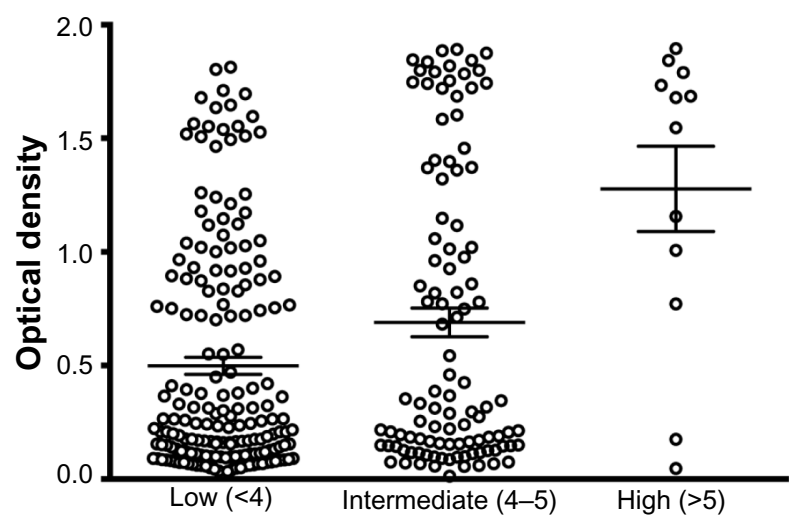

Figure 2 Correlation between patient 4T score and the optical density (OD) of anti-heparin/platelet factor 4 enzyme-linked immunosorbent assays.

Notes: (A) Boxplot representing the 4T scores of negative (OD $<0.7$ ), lowpositive $(0.7 \leq O D \leq 1.4)$, and high-positive $(O D>1.4)$ groups. Error bars represent the minimum and maximum values. Statistical significance $\left(^{*}\right)$ was calculated using two-way analysis of variance $(P<0.05)$. (B) Scatterplot representing OD of low $(4 \mathrm{~T}$ score $<4)$, intermediate $(4 \mathrm{~T}$ score $=4-5)$, and high $(4 \mathrm{~T}$ score $>5$ ) pretest probability groups. Mean OD and the standard error of the mean are represented by the horizontal error bars.

\section{Discussion}

Our study offers insight to the clinical characteristics of patients receiving PF4 ELISAs. Many of the clinical and laboratory characteristics that were significantly different when comparing the patients with positive and negative PF4 ELISA tests were no longer significantly different when only those with low-positive PF4 ELISA OD values $(<1.4)$ were used. In contrast, we found that patients with high OD positivity $(>1.4)$ represent a distinct clinical group when compared with the negative group. Not only did they have a larger drop in platelet count from admission but they also had a higher white blood cell count and more antibiotic usage. In addition, the high-positive PF4 ELISA group not only had higher 4T scores than the negative assay group, but also significantly higher than the low-positive PF4 ELISA group. These findings suggest that the low-positive group encompasses a clinically heterogeneous population of patients who are difficult to distinguish clinically. Because there is no gold standard for a HIT diagnosis, we cannot confidently state that our data support the hypothesis that a high PF4 ELISA OD associates with a diagnosis of HIT. However, we did find that those patients with a high PF4 ELISA OD represent a distinct clinical group. Our findings also reinforce the clinical utility of the 4T score, although those patients with a low-positive PF4 ELISA OD had a significantly higher 4T score than those patients with negative assay results.

Like previous studies, our patient population demonstrated no differences between the likelihood of HIT and the primary treatment team. ${ }^{40} \mathrm{We}$ also observed a direct correlation between OD positivity and $4 \mathrm{~T}$ score, as previously reported. ${ }^{6,17,19,20,31,40}$ While no statistically significant differences in mortality were found in our cohort, there was a trend toward decreased mortality in patients with higher OD positivity, which has also been previously observed. ${ }^{23}$ Although counterintuitive, this finding has been attributed to the possible presence of other, more life-threatening conditions that may be causing thrombocytopenia in patients with lower OD positivity. While these previous studies have focused on narrow cohorts of patients from specific services or single institutions, our analysis includes patients spanning several services from institutions with vastly different resources. Our study additionally characterizes hospitalized patients with both negative and positive OD with thresholds that more accurately reflect those used in clinical practice. We, therefore, believe the findings from our study are widely generalizable.

The retrospective nature of our study introduces inherent limitations, including differences in the timing of medical decisions by the primary team and biases when 
calculating $4 \mathrm{~T}$ scores. To mitigate hindsight bias, we recorded the primary team's assessment at the time the assay was collected when available. The lack of a definitive diagnosis of HIT reflects the limitations of currently available diagnostic tools. While the SRA is commonly accepted as a more specific test compared with the PF4 ELISA, the test is technically demanding which limits its accessibility. In fact, only a small proportion of patients in our study received SRA, and, while statistical significance was not achieved likely due to the small sample size, patients with positive SRA generally had higher OD, as has been previously described (data not shown). ${ }^{6,18-20}$ It has recently been shown that the diagnostic accuracy of PF4 ELISA can be significantly improved by increasing the threshold defining positive tests. ${ }^{41,42}$ Our data suggest that these more stringent thresholds associate with clinical and laboratory groups.

\section{Conclusion}

In summary, this study characterizes hospitalized patients receiving PF4 ELISA at two large, urban academic institutions and found that patients with high-positive ODs represent a distinct clinical group when compared with low-positive ODs. However, no single characteristic we analyzed was able to distinguish those with a low-positive PF4 ELISA OD and those with a high-positive PF4 ELISA OD. Our findings reinforce the clinical utility of the 4T score, which was the only clinical variable that significantly distinguished low-positive and highpositive groups. Absence of thrombosis and temporal onset of thrombocytopenia consistent with HIT, important components of the 4T score, were commonly observed in those both negative and low-positive PF4 ELISA ODs unlikely to represent true HIT, and thus these assays, ordered on both medical and surgical services (Figure 1A), were likely not appropriate. While HIT remains a challenging clinicopathologic diagnosis for clinicians, further clinical and molecular characterization of high-positive PF4 ELISA OD patients will improve our ability to accurately diagnose this disease.

\section{Acknowledgments}

The authors would like to thank Michael A Nardi, MS and the Bellevue Hospital Center Hematology Laboratory for their assistance in identifying, testing, and entering the sample results into the database referenced in this work. We also thank the anonymous reviewers for suggestions.

\section{Disclosure}

The authors report no conflicts of interest in this work.

\section{References}

1. Girolami B, Prandoni P, Stefani PM, et al. The incidence of heparininduced thrombocytopenia in hospitalized medical patients treated with subcutaneous unfractionated heparin: a prospective cohort study. Blood. 2003;101:2955-2959.

2. Pouplard C, May MA, Regina S, Marchand M, Fusciardi J, Gruel Y. Changes in platelet count after cardiac surgery can effectively predict the development of pathogenic heparin-dependent antibodies. $\mathrm{Br} J$ Haematol. 2005;128:837-841.

3. Warkentin TE, Levine MN, Hirsh J, et al. Heparin-induced thrombocytopenia in patients treated with low-molecular-weight heparin or unfractionated heparin. N Engl J Med. 1995;332:1330-1335.

4. Martel N, Lee J, Wells PS. Risk for heparin-induced thrombocytopenia with unfractionated and low-molecular-weight heparin thromboprophylaxis: a meta-analysis. Blood. 2005;106:2710-2715.

5. Warkentin TE. Heparin-induced thrombocytopenia: pathogenesis and management. Br J Haematol. 2003;121:535-555.

6. Arepally GM, Ortel TL. Clinical practice. Heparin-induced thrombocytopenia. N Engl J Med. 2006;355:809-817.

7. Lo GK, Juhl D, Warkentin TE, Sigouin CS, Eichler P, Greinacher A. Evaluation of pretest clinical score (4 T's) for the diagnosis of heparininduced thrombocytopenia in two clinical settings. J Thromb Haemost. 2006;4:759-765.

8. Warkentin TE, Heddle NM. Laboratory diagnosis of immune heparininduced thrombocytopenia. Curr Hematol Rep. 2003;2:148-157.

9. Linkins LA, Bates SM, Lee AYY, Heddle NM, Wang G, Warkentin TE. Combination of 4Ts score and PF4/H-PaGIA for diagnosis and management of heparin-induced thrombocytopenia: prospective cohort study. Blood. 2015;126:597-603.

10. Warkentin TE, Linkins LA. Non-necrotizing heparin-induced skin lesions and the 4T's score. J Thromb Haemost. 2010;8:1483-1485.

11. Fiorenza MA, Frazee EN, Personett HA, Dierkhising RA, Schramm GE. Assessment of a modified 4T scoring system for heparin-induced thrombocytopenia in critically ill patients. J Crit Care. 2014;29:426-431.

12. Cuker A, Gimotty PA, Crowther MA, Warkentin TE. Predictive value of the 4Ts scoring system for heparin-induced thrombocytopenia: a systematic review and meta-analysis. Blood. 2012;120:4160-4167.

13. Kelton JG, Smith JW, Warkentin TE, Hayward CP, Denomme GA, Horsewood P. Immunoglobulin G from patients with heparin-induced thrombocytopenia binds to a complex of heparin and platelet factor 4 . Blood. 1994;83:3232-3239.

14. Visentin GP, Ford SE, Scott JP, Aster RH. Antibodies from patients with heparin-induced thrombocytopenia/thrombosis are specific for platelet factor 4 complexed with heparin or bound to endothelial cells. J Clin Invest. 1994;93:81-88.

15. Greinacher A, Pötzsch B, Amiral J, Dummel V, Eichner A, Mueller-Eckhardt C. Heparin-associated thrombocytopenia: isolation of the antibody and characterization of a multimolecular PF4-heparin complex as the major antigen. Thromb Haemost. 1994;71:247-251.

16. Amiral J, Bridey F, Dreyfus M, et al. Platelet factor 4 complexed to heparin is the target for antibodies generated in heparin-induced thrombocytopenia. Thromb Haemost. 1992;68:95-96.

17. Warkentin TE, Greinacher A, Koster A, Lincoff AM. Treatment and prevention of heparin-induced thrombocytopenia: American College of Chest Physicians Evidence-Based Clinical Practice Guidelines (8th Edition). Chest. 2008;133:340S-380S.

18. Warkentin TE, Sheppard J, Moore JC, Sigouin CS, Kelton JG. Quantitative interpretation of optical density measurements using PF4-dependent enzyme-immunoassays. J Thromb Haemost. 2008;6:1304-1312.

19. Pouplard C, Amiral J, Borg JY, Laporte-Simitsidis S, Delahousse B, Gruel Y. Decision analysis for use of platelet aggregation test, carbon 14-serotonin release assay, and heparin-platelet factor 4 enzyme-linked immunosorbent assay for diagnosis of heparin-induced thrombocytopenia. Am J Clin Pathol. 1999;111:700-706.

20. Verma AK, Levine M, Shalansky SJ, Carter CJ, Kelton JG. Frequency of heparin-induced thrombocytopenia in critical care patients. Pharmacotherapy. 2003;23:745-753. 
21. Warkentin TE. How I diagnose and manage HIT. Hematology Am Soc Hematol Educ Program. 2011;2011:143-149.

22. Warkentin TE, Sheppard JA, Horsewood P, Simpson PJ, Moore JC, Kelton JG. Impact of the patient population on the risk for heparininduced thrombocytopenia. Blood. 2000;96:1703-1708.

23. Lo GK, Sigouin CS, Warkentin TE. What is the potential for overdiagnosis of heparin-induced thrombocytopenia? Am J Hematol. 2007;82 1037-1043.

24. Martinuzzo ME, Forastiero RR, Adamczuk Y, Pombo G, Carreras LO. Antiplatelet factor 4 - heparin antibodies in patients with antiphospholipid antibodies. Thromb Res. 1999;95:271-279.

25. Schenk S, El-Banayosy A, Prohaska W, et al. Heparin-induced thrombocytopenia in patients receiving mechanical circulatory support J Thorac Cardiovasc Surg. 2006;131:1373-1381. e4.

26. Amiral J, Peynaud-Debayle E, Wolf M, Bridey F, Vissac AM, Meyer D. Generation of antibodies to heparin-PF4 complexes without thrombocytopenia in patients treated with unfractionated or low-molecular-weight heparin. Am J Hematol. 1996;52:90-95.

27. Arepally GM, Ortel TL. Heparin-induced thrombocytopenia. Annu Rev Med. 2010;61:77-90.

28. Zwicker JI, Uhl L, Huang WY, Shaz BH, Bauer KA. Thrombosis and ELISA optical density values in hospitalized patients with heparininduced thrombocytopenia. J Thromb Haemost. 2004;2:2133-2137.

29. Baroletti S, Hurwitz S, Conti NA, Fanikos J, Piazza G, Goldhaber SZ. Thrombosis in suspected heparin-induced thrombocytopenia occurs more often with high antibody levels. Am J Med. 2012;125:44-49.

30. Greinacher, Ittermann, Gemühl, et al. Heparin-induced thrombocytopenia: towards standardization of platelet factor $4 /$ heparin antigen tests. J Thromb Haemost. 2010;8:2025-2031.

31. Janatpour KA, Gosselin RC, Dager WE, et al. Usefulness of optical density values from heparin-platelet factor 4 antibody testing and probability scoring models to diagnose heparin-induced thrombocytopenia. Am J Clin Pathol. 2007;127:429-433.

32. Rhodes GR, Dixon RH, Silver D. Heparin induced thrombocytopenia with thrombotic and hemorrhagic manifestations. Surg Gynecol Obstet. 1973;136:409-416.
33. Babcock RB, Dumper CW, Scharfman WB. Heparin-induced immune thrombocytopenia. N Engl J Med. 1976;295:237-241.

34. Vanderschueren S, De Weerdt A, Malbrain M, et al. Thrombocytopenia and prognosis in intensive care. Crit Care Med. 2000;28:1871-1876.

35. Levine MN, Hirsh J, Gent M, et al. Prevention of deep vein thrombosis after elective hip surgery. A randomized trial comparing low molecular weight heparin with standard unfractionated heparin. Ann Intern Med. 1991;114:545-551.

36. Warkentin TE, Kelton JG. Heparin-induced thrombocytopenia. Аnnu Rev Med. 1989;40:31-44.

37. Warkentin TE. Heparin-induced thrombocytopenia in critically ill patients. Crit Care Clin. 2011;27:805-823, v.

38. Smythe MA, Warkentin TE, Woodhouse AL, Zakalik D. Venous limb gangrene and fatal hemorrhage: adverse consequences of HIT "overdiagnosis" in a patient with antiphospholipid syndrome. Am J Hematol. 2011;86:188-1891.

39. Whitlatch NL, Perry SL, Ortel TL. Anti-heparin/platelet factor 4 antibody optical density values and the confirmatory procedure in the diagnosis of heparin-induced thrombocytopenia. Thromb Haemost. 2008;100:678-684.

40. Pearson MA, Nadeau C, Blais N. Correlation of ELISA optical density with clinical diagnosis of heparin-induced thrombocytopenia: a retrospective study of 104 patients with positive anti-PF4/heparin ELISA. Clin Appl Thromb Hemost. 2014;20:349-354.

41. Raschke RA, Curry SC, Warkentin TE, Gerkin RD. Improving clinical interpretation of the anti-platelet factor 4/heparin enzymelinked immunosorbent assay for the diagnosis of heparin-induced thrombocytopenia through the use of receiver operating characteristic analysis, stratum-specific likelihood ratios, and Bayes theorem. Chest. 2013;144:1269-1275.

42. Chan CM, Woods CJ, Warkentin TE, Sheppard J-AIA, Shorr AF. The role for optical density in heparin-induced thrombocytopenia: a cohort study. Chest. 2015;148:55-61.
Journal of Blood Medicine

\section{Publish your work in this journal}

The Journal of Blood Medicine is an international, peer-reviewed, open access, online journal publishing laboratory, experimental and clinical aspects of all topics pertaining to blood based medicine including but not limited to: Transfusion Medicine; Blood collection, Donor issues, Transmittable diseases, and Blood banking logistics; Immunohematology; Artificial and alternative

\section{Dovepress}

blood based therapeutics; Hematology; Biotechnology/nanotechnology of blood related medicine; Legal aspects of blood medicine; Historical perspectives. The manuscript management system is completely online and includes a very quick and fair peer-review system. Visit http://www.dovepress.com/ testimonials.php to read real quotes from published authors. 